\title{
Erosion Wear on Impeller of Double-Suction Centrifugal Pump due to Sediment Flow
}

\author{
Z. Shen ${ }^{\dagger}$, R. Li, W. Han, H. Quan and R. Guo \\ School of Energy and Power Engineering, Lanzhou University of Technology, Lanzhou, Gansu, China \\ Key Laboratory of Fluid Machinery and Systems of Gansu Province, Lanzhou Gansu, China \\ †Corresponding Author Email: szj007cool@126.com
}

(Received August 28, 2019; accepted November 26, 2019)

\begin{abstract}
Erosion wear of the centrifugal pump impeller are the key issues since its operating in sediment-laden flow. Experiment and numerical method are adopted in this study to investigate the erosion wear of the impeller in a double-suction pump in the Jingtai Yellow River Irrigation Project (JYRIP). Observation during operation combined with morphology study is carried out to verify erosion mechanisms at the different sections of the impeller blade. For numerical study, the Eulerian-Lagrangian approach combined with the Mclaury model is employed to predict the trajectories of the particles under different particle parameters. Also the effects of particle size and concentration on impeller erosion wear is studied. Numerical results are consistent with experimental data. The surface wear of the blade changes obviously from circular shape to strip shape in the direction from the Leading Edge (LE) to the Trailing Edge (TE), and the wear damage area increases correspondingly. Trajectories of larger particles are more uniform and tend to cause more severe erosion wear damage. The wear damage tends to be slow after the particle size increases to a certain extent under the same sediment concentration. Besides, erosion rate increases with an increase in sediment concentration, and the erosion rate of the suction side is greater than the pressure side at the same position of the blade surface. These results can offer a good reference for the design of wear resistance impeller of the double suction pump.
\end{abstract}

Keywords: Double-suction centrifugal pump; Two phase flow; Erosion wear; Particle parameters.

\section{NOMENCLATURE}

$e_{\mathrm{N}}$ normal restitution coefficient

$e_{\mathrm{T}} \quad$ tangent restitution coefficient

$f_{\mathrm{i}} \quad$ all the external force acting on the liquid phase

$F_{\text {others }}$ all the other forces acting on the particle

$F S \quad$ shape factor of sediment particles

$H$ head

$P \quad$ pressure of the liquid phase

$Q_{d} \quad$ pump design flow rate

$r_{\mathrm{p}} \quad$ volume fraction of particle

$r_{f} \quad$ volume fraction of fluid

$u \quad$ velocity of the liquid phase

$u_{\mathrm{p}} \quad$ velocity of the particle

$V \quad$ velocity of the sand particle

\section{Greek Symbols}

$\beta \quad$ particle mass loading

$\varepsilon_{H} \quad$ relative errors of pump head

$\varepsilon_{\eta} \quad$ relative errors of pump efficiency

$\eta \quad$ efficiency

$\theta \quad$ impact angle

$\rho \quad$ density of the liquid phase

$\rho_{\mathrm{p}} \quad$ density of particles $\tau_{r} \quad$ drag force related to the angular particle

$v \quad$ kinetic viscosity

$\begin{array}{ll}\text { Subscripts } \\ f & \text { fluid } \\ \mathrm{N} & \text { normal } \\ p & \text { particles } \\ r & \text { ratio } \\ \mathrm{T} & \text { tangent }\end{array}$

\section{Abbreviations}

DPM Discrete Phase Model

DRW Discrete Random Walk

FESEM Field Emission Scanning Electron

Microscope

JYRIP Jingtai Yellow River Irrigation Project

LE Leading Edge

PS Pressure Side

SEM Scanning Electron Microscopy

SS Suction Side

SST Shear Stress Transport

TE Trailing Edge 


\section{INTRODUCTION}

Double-suction centrifugal pumps are widely used in agricultural water conservancy projects due to their high reliability and high capacity. JYRIP is the first high-lift, large-capacity multi-level irrigation project in Gansu, China. The establishment of the project has greatly improved the local agricultural irrigation environment and achieved obvious economic benefits. However, the sediment concentration in the Yellow River is very high. The presence of sediment particles during the pumping process directly affects the internal flow of the pump, causing severe wear of the impeller and pump casing, resulting in a reduction in pump performance and low reliability, and a shortened cycle of overflow components.

Although the hardness and compressive strength of blades can be improved by coating protection method, delamination fracture often occurs due to the fact that the impeller works in water for a long time and the adhesion of the coating is not greatly improved. The fundamental way to solve the erosion wear problem is to establish the relationship between particle parameters and wear. The relationship between particle parameters and erosion wear has been widely investigated in recent years (Nguyen et al. 2016; Parsi et al. 2017; Liu et al. 2014).

However, due to the complexity of the structure of the double-suction centrifugal pump, the quantitative correlation of the mechanism of impeller blade wear is lack of systematic research. The morphology observation experiment method can locate the erosion wear form and damage degree of the blade surfaces after running for a period of time (Serrano et al. 2018; Chitrakar et al. 2016). Additionally, to optimize the design of doublesuction impeller and the pumping system, it is important to consider the influence of particles on the trajectories in the computational domain and identify the location and erosion rate density distribution on the impeller blades for sediment flows in pumps. Computational Fluid Dynamics (CFD) combined with appropriate erosion modeling has many advantages that can be used to predict erosion damage in any complex computational domain. Many empirical erosion models have been developed to predict the erosion in the sand laden flow. Their erosion all include the impingement angle, impact velocity, particle diameter, particle mass, and collision frequency plugged et al.

Some of the more classic models include the one proposed by Finnie (1960), this model is more suitable for ductile materials, where the erosion varies with the particle velocity and impact angle. As a deformed model, Bitter (1963), can be used at higher impact angle near $90^{\circ}$. This erosion model indicates that the maximum erosion damage occurs at lower impact angles for ductile materials and higher impact angles near to $90^{\circ}$ for the brittle materials. Then, Hashish (1988) modified the model for low impact angles in the range of $0^{\circ}$ to $40^{\circ}$ of particles with high density on ductile materials. To evaluate the empirical constants in the equations derived by Finnie, McLaury (1996) and Ahlert
(1994) at the E/CRC of the University of Tulsa performed a series of erosion tests and proposed the erosion model which can be adopted to predict the erosion wear of sediment water in many fields. In the erosion model proposed by Oka (2005), empirical constants of velocity and diameter were also included in the equations, it provides a more realist correlation by containing the effect of wall material hardness. (Haiwen zhu et al. 2019). designed an electrical submersible pump (ESP) testbed to study the sand particles erosion mechanism and validated the numerical simulation results by embedding different erosion models in the fluid dynamics software.

In this research, the erosion wear of an impeller in a double-suction pump in JYRIP was investigated by experiment and numerical method. Regular operation observation combined with morphology study of erosion wear of the impeller were carried out to study the erosion wear mechanism on the different positions of the pressure side and suction side. The blade was cut from the impeller by plasma cutting. In addition, the effects of particle size and sediment particle concentration on the particle trajectory and erosion wear characteristics in the computational domain were studied.

\section{GEOMETRIC MODEL}

The research object of this paper is taken from a pumping station of the JYRIP. The double-suction centrifugal pump is mainly composed of pump casing, an impeller, impeller ring and sealing as shown in Fig.1. And Fig.2 shows the schematic of double-suction impeller, as can be seen, the double suction impeller is a symmetrical combination of two single-suction impellers.

The design parameters of the pump are as follows: the design flow rate $Q_{\mathrm{d}}$ is $350 \mathrm{~L} / \mathrm{s}$, the head $H$ is 43.5 , the efficiency $\eta$ is $88.84 \%$, the shaft speed of $n$ is $1450 \mathrm{r} / \mathrm{min}$, the impeller eye diameter and impeller outlet diameter is $350 \mathrm{~mm}$ and $406 \mathrm{~mm}$ respectively. The number of blades is designed to be 6 . Specific speed is a quasi non-dimensional number which generally used in pump productions to categorizes different type of pump impellers. For double-suction centrifugal pump, the definition of specific speed can be described as following:

$n_{s}=\frac{3.65 n \sqrt{Q_{d} / 2}}{H^{3 / 4}}$

According to the equation (1), the $n_{\mathrm{s}}$ of impeller of this pump is 130.6 .

Allowable operating region flow rates should be determined at first to avoid pump running under low reliability. Pump running under lower or higher flow rates both impacts the flow passages and results in flow separation and cavitation. According to the inlet velocity triangle of the impeller, shockless entry near the inlet of impeller will occur only if the correct blade inlet angle $\beta_{1}$. In this study, the blade inlet edge were assumed to be parallel to the flow relative velocity, and the shockless capacity could be $416 \mathrm{~L} / \mathrm{s}$ according to the equation offered by Van (2015). 


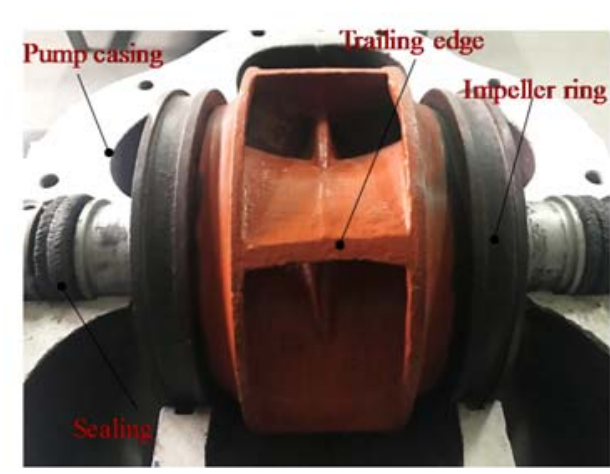

Fig. 1. The prototype of the double-suction centrifugal pump.

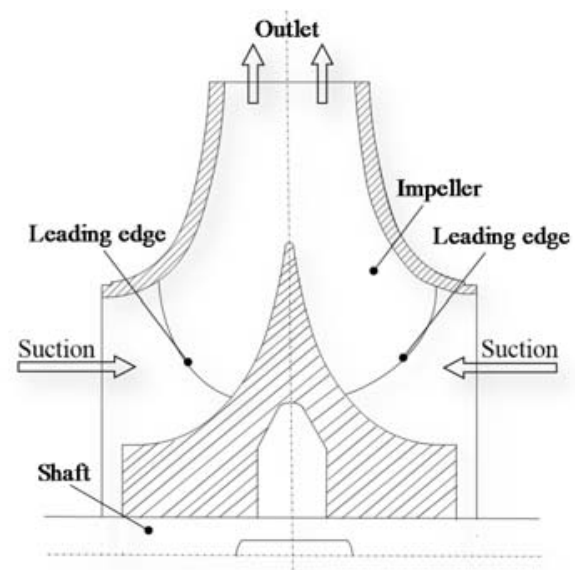

Fig. 2. The schematic of double-suction impeller.

\section{EXPERIMENTAL STUDY OF THE SEDIMENT EROSION OF THE IMPELLER}

The erosive wear of the impeller surface occurs when particles impact with the surface at a certain velocity. The surfaces of the eroded impeller blades are investigated by regular operation observation and the morphology study. The impeller was made of QT600-3 ductile cast iron, and the material properties of the impeller are shown in Table 1.

Table 1 material properties of the impeller

\begin{tabular}{|c|c|}
\hline Property & Value \\
\hline Density $\left(\mathrm{kg} / \mathrm{m}^{3}\right)$ & 7300 \\
\hline Hardness $(\mathrm{HB})$ & $229-302$ \\
\hline Tensile strength(Mpa) & 600 \\
\hline Yield strength $(\mathrm{Mpa})$ & 420 \\
\hline
\end{tabular}

\subsection{Regular Operation Observation}

According to the regular operating data by $\mathrm{Lu}$ (1999), obvious erosion wear damages appear on the blade surfaces after running for $3,000 \mathrm{~h}$. And abnormal vibrations occur after running for 10,000 $\mathrm{h}$, the impeller blades were severely worn, pits with a depth of $3.0 \mathrm{~mm}$ to $4.5 \mathrm{~mm}$ and an area of 500 square millimeters appeared near the TE (Zhao 2009). The change of impeller surface appearance leads to the more complex flow structure inside the pump and the increase of disorder pressure fluctuation (Bai et al. 2019; Quan et al. 2017).

In this study, the impeller surfaces morphologies were observed every $1,000 \mathrm{~h}$ during the regular operation of the pumping irrigation as shown in Table 2. Significant erosion wear damages appear after running 2,000 h. After that, the blade damage increased and it was completely destroyed after running 8,000 hours, this will result in the intensification of vibration and noise of pump system in the operation. Figure 3 shows the eroded impeller with holes after running 7,000 $\mathrm{h}$. It can be shown that the location of the eroded holes are mostly concentrated near the leading edge of the blades. The cause of this result may is the combined effect of erosion and cavitation. Many evidences show that the damage of cavitation at the impeller inlet is directly related to the flow recirculation at the impeller suction (Hirschi et al. 1998; CoutierDelgosha et al. 2003). However, cavitation damage and erosion damage of the surfaces of impeller are difficult to be distinguished from numerical results, so we decided to consider only the single effect of erosion in the numerical calculation. In our numerical analysis in this study, all cases were run at the best efficiency flow condition.

Table 2 Regular operation observation of impeller

\begin{tabular}{|c|c|}
\hline Time & Erosion wear morphology \\
\hline $1,000 \mathrm{~h}$ & Not obvious \\
\hline $2,000 \mathrm{~h}$ & Pits and debris appear \\
\hline $3,000 \mathrm{~h}$ & Severe damages appear \\
\hline $5,000 \mathrm{~h}$ & All surfaces were eroded with pits \\
\hline $8,000 \mathrm{~h}$ & All surfaces were eroded with holes \\
\hline
\end{tabular}

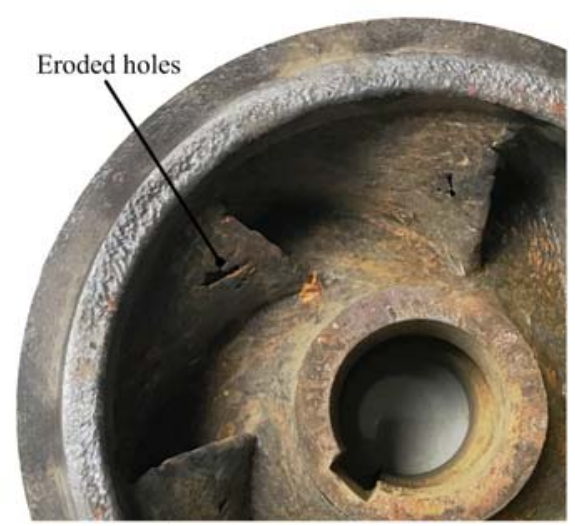

Fig. 3. Eroded impeller with holes after running $7000 \mathrm{~h}$.

\subsection{Morphology Study of Erosion Wear}

The wear morphology of the blade surface is related to the contact characteristics of the particles with the blade surface. Different particle impact angle and 
impact velocity lead to different blade surface wear profiles. Wilson et al. (2006) claimed that sliding and impact erosion wear are the main forms in pumps depend on the impact angle of particles. In addition, Stachowiak (2013) summarized 4 different mechanisms for sediment erosion in hydraulic machinery, including surface fatigue, brittle fracture, abrasive erosion and plastic deformation.

To investigate the erosion characteristics of the impeller, an impeller running for 5,000 $\mathrm{h}$ was taken as the research object. One of the blades was cut from the impeller by plasma cutting and divided into eight sections. Figure 4 shows the schematic of blade cutting. As an effective analytical tool for morphology research, scanning electron microscopy (SEM) can be used to obtain the microscopic structure of specimens at a high resolution. The field emission scanning electron microscope (Quanta FEG 450) was used to image the blade surfaces morphologies in this study. In order to increase the accuracy of the test, reduce other influencing factors, the surface of the tested samples cut from the eroded impeller blade had been polished first, and dried in an ultrasonic cleaner containing ethanol solution.

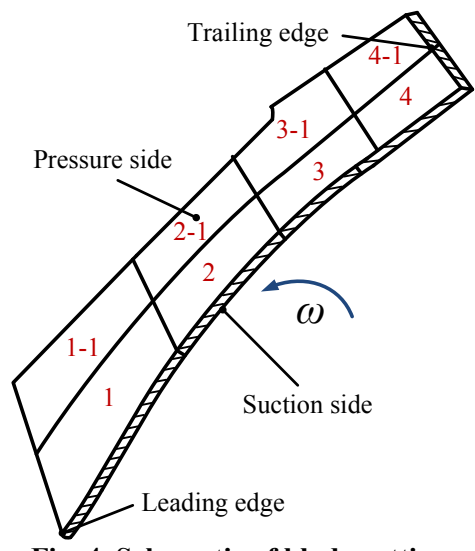

Fig. 4. Schematic of blade cutting.

Figure 5 shows the pressure side of the eroded blade. As shown in Fig.5 (a) and Fig.5 (e), remarkable grooves and pits were observed on the sample 1-1 and sample 1 near the blade LE. And the shapes of the grooves are irregular and distorted. Because the flow in this area is relatively disordered and sediment particles impact the LE at low velocity and high impact angle when entering in the inlet of the impeller. Figure 5 (b) and Fig.6 (5) show that more pits appeared on the surfaces of sample 2-1 and sample 2, and the pits in this area are deeper than sample 1-1 and sample 1. In this region, the sediment particles obtain a certain kinetic energy under the rotation of the blade, and impact with the blade at a greater velocity. Furthermore, on the middle parts of the blade surface, under the continuous impact of particles with small angular velocity, the shape of the pits changes from a circular shape to a strip shape as show in Fig.5 (c) and Fig.5 (g). However, remarkable strip shape damages are showed in Fig.5 (d) and Fig.5 (h) on the surfaces of sample 4-1 and 4 near the TE, and the strips mainly in the direction of the mainstream, the abrasive/cutting effects are dominant in this area. The blade TE was worn in a zigzag shape under the continuous impact of the sediment particles.

Figure 6 shows the SEM morphologies of surfaces sample 2-1. It can be seen that debris piled up and cracks distributed around the pits in this region for the reason of medium velocity and higher impact angle of the sediment particles. This is a typical combination of fatigue erosion wear and plastic deformation according to Stachowiak and Batchelor (2013).

Figure 7 shows the wear morphology of the suction side of the blade. The samples near the LE were found to be severely eroded with large radius pits and strip shape damages as shown in Fig.7 (a) and Fig.7 (e). The erode damage on the suction side of the sample 1-1 and sample 1 are severer than the same position of the pressure side. Due to the sudden change in the direction of flow after the sediment particles enter in the impeller, flow separation occurs on the suction side of the blade inlet, causing flow disturbances in this region. The erosion pattern of other areas on the suction side of the blade are similar to that on the pressure side of the blade. However, compared with the blade TE of pressure side, the wear degree of suction surface at the same position is smaller as shown in Fig. 7(d) and Fig.7 (h).

\section{NUMERICAL STUDY}

The methodological approach taken in this research is a mixed methodology based on EulerianLagrangian coordinate system. Liquid phase is expressed using Navier-Stokes equations in the Eulerian framework and the solid phase is calculated by tracking the trajectories of particles based on the Tabakoff particle rebound model (Grant and Tabakoff 1975). The erosion wear distribution and erosion rate caused by particle impacting on the blade surface is predicted using Mclaury erosion model. Numerical study is performed for different sediment particles diameters in the solid-liquid phase flow to investigate the effects of particle diameter on erosion wear damage. Details of the numerical methodology is described below.

\subsection{Liquid Phase Equation}

The liquid phase water in this study is assumed as incompressible, and there is no heat exchange between liquid phase and solid phase. So the continuity equation and momentum equations of the liquid phase can be described as following (Shen et al. 2019):

$\nabla \cdot u=0$

$\frac{\partial u}{\partial t}+u \cdot \nabla u=-\frac{1}{\rho} \nabla P+v \nabla^{2} u+\frac{1}{3} v \nabla(\nabla \cdot u)+f_{i}(3)$

Where $u, \rho$ and $P$ are the velocity, density and pressure of the liquid phase, $v$ is the kinetic viscosity and $f_{\mathrm{i}}$ is the external force acting on the liquid phase. 


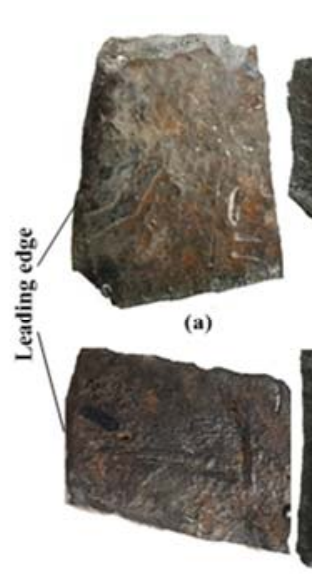

(e)

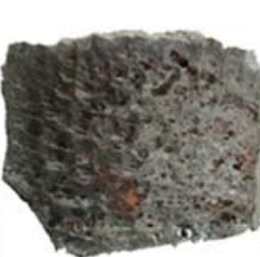

(b)

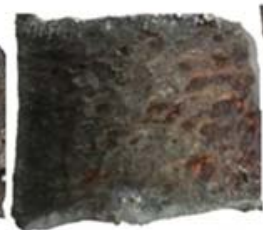

(f)

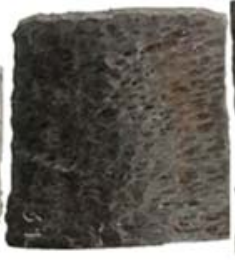

(c)

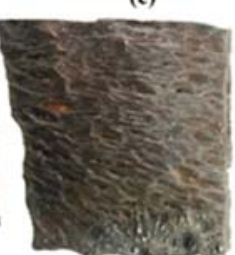

(g)

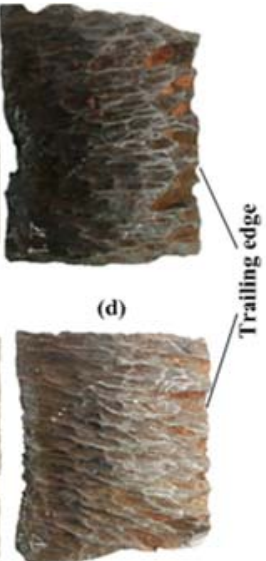

(h)

Fig.5. Morphologies of pressure side(PS) surfaces after running $5000 \mathrm{~h}$. (a) Sample 1-1 (b) Sample 2-1 (c) Sample 3-1 (d) Sample 4-1 (e) Sample 1 (f) Sample 2(g) Sample 3 (h) Sample 4.

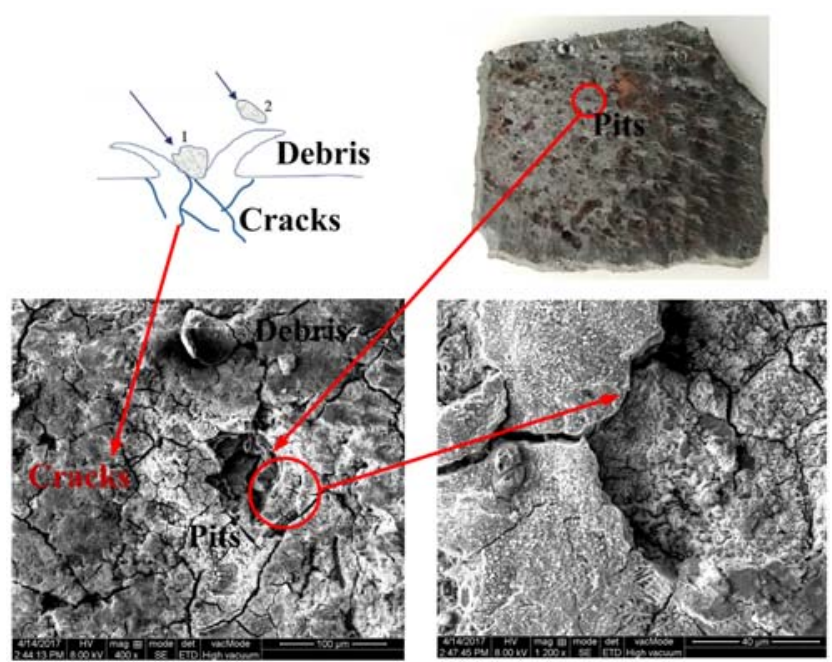

Fig. 6. SEM morphologies of surfaces sample 2-1.

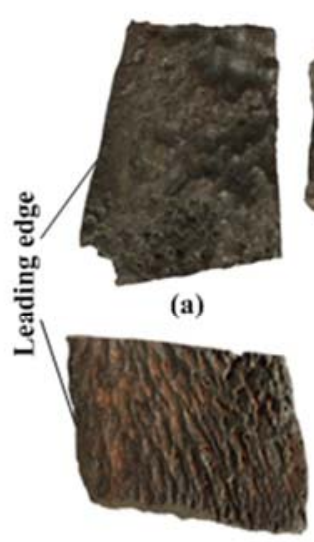

(e)

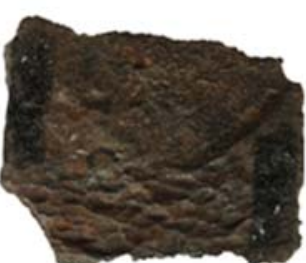

(b)

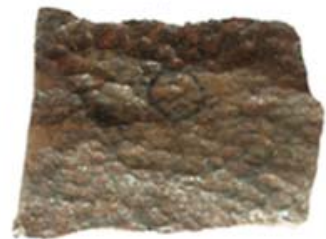

(f)

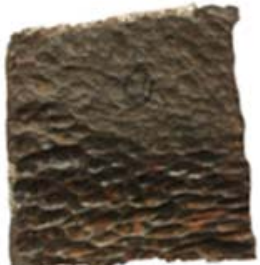

(c)

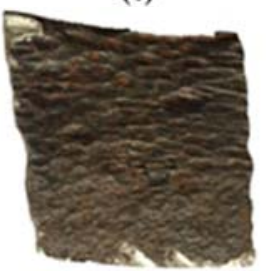

(g)

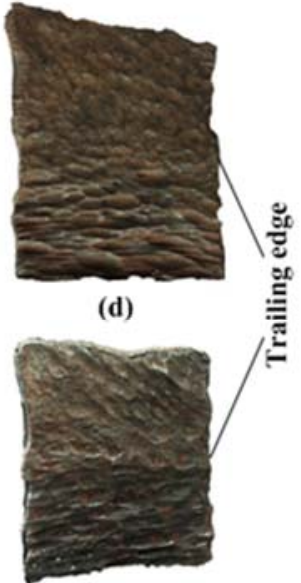

(h)

Fig. 7. Morphologies of suction side(SS) surfaces after running $5000 \mathrm{~h}$.

(a) Sample 1-1 (b) Sample 2-1 (c) Sample 3-1 (d) Sample 4-1. 
The k-omega Shear Stress Transport (SST) turbulent model is also used in this study to investigate the flow of liquid phase (Wang et al. 2019). This model can not only calculate the flow in the core region, but also capture the details of boundary layer flow (ANSYS FLUENT 12.0/12.1 Documentation; Menter, 1994).

\subsection{Solid Phase Equation}

In this study, the sediment particles are considered as discrete phase in the numerical simulation, so Discrete Phase Model (DPM) is adopted to track the motion of sand particles. The particle velocity can be calculated as the following:

$\frac{d u_{\mathrm{p}}}{d t}=\frac{u-u_{\mathrm{p}}}{\tau_{r}}+\frac{g\left(\rho_{\mathrm{p}}-\rho\right)}{\rho_{\mathrm{p}}}+F_{\text {others }}$

Where $u$ is the velocity of the liquid phase, $u_{\mathrm{p}}$ is the velocity of the particle, $\rho_{\mathrm{p}}$ is the density of particles and $F_{\text {others }}$ is all the other forces acting on the particle, $\tau_{r}$ is related to the drag force which is evaluated by the empirical formulation for angular particle developed by Haider and Levenspiel (1989) .

The particle mass loading, $\beta$ should be calculated first to determine which way interacted between liquid phase and solid phase. According to Brown (2002), two-way coupling should be used in the calculation wen the particle mass loading $\beta$ is greater than 0.2 . In this study, the value of $\beta$ is less than 0.2 according to the sediment particles concentration date of the JYRIP, so one-way coupling is used in this study. The $\beta$ is expressed as the following:

$\beta=\frac{r_{p} \rho_{p}}{r_{f} \rho_{f}}$

Where $r_{\mathrm{p}}$ and $r_{f}$ are the volume fraction of particle and fluid respectively, $\rho_{p}$ and $\rho_{f}$ is the density of particle and fluid.

\subsection{Particle Impact and Rebound Model}

To calculate the sediment particles trajectories in the computational domain, the collision process between particles and wall must be calculated. The stochastic model developed by Wakeman and Tabakoff (1982) is adopted in this work to solve the direction and velocity of particles after impact with solid wall:

$e_{\mathrm{N}}=1.0-0.0211 \theta+0.00228 \theta^{2}-0.000000876 \theta^{3}$

$e_{\mathrm{T}}=0.953-0.000446 \theta+0.00000648 \theta^{3}(7)$

Where $e_{\mathrm{N}}$ and $e_{\mathrm{T}}$ are the normal and tangent restitution coefficient, $\theta$ is the impact angle.

\subsection{Erosion Model}

Many empirical erosion models have been published in the previous literature. All include the impingement angle, impact velocity, particle diameter, particle mass, and frequency of impact plugged into it. The impact factors among these three models have already been discussed in many previous studies. Regardless of which of the above models, the flow passage components wall, the impact of the particles and the erosion wear caused by the friction are complex functions related to environmental factors, particle properties and target properties.

As the first person to propose an erosion model, Finnie (1960) expressed the model as:

$E=k v_{p}^{n} f(\theta)$

$f(\theta)=\frac{1}{3} \cos ^{2} \theta \quad$ for $\theta>18.5^{\circ}$

$f(\theta)=\sin (2 \theta)-3 \sin ^{2} \theta \quad$ for $\theta \leq 18.5^{\circ}$

Where $\mathrm{k}$ is an empirical coefficient, $v_{p}$ is the particle impact velocity, and $f(\theta)$ is a function of the impact angle $\theta$. The value of the exponent $\mathrm{n}$ is generally in the range 2.3 to 2.5 radians. The default constants for the ANSYS Fluent Finnie Erosion model are only valid for erosion calculations where sand particles hit carbon steel walls.

To determine the empirical constants above the equation derived by Finnie, McLaury (1996) and Ahlert (1994) performed a series of erosion tests and developed a new model to predict erosion for multiphase flow, the erosion rate per unit area can be expressed as:

$E=A F_{S} V^{n} f(\theta)$

Where $A$ is $1.997 \mathrm{e}^{-7}$ for the empirical constant, $F_{\mathrm{s}}$ is the shape factor of sediment particles, which is 1.0 for angular, 0.53 for semirounded, and 0.2 for spherical particles. $n$ is also an empirical constant, the value of 1.73 was adopted in this study, $V$ is the velocity of the sand particle. $f(\theta)$ is the impact function relate to the impact angle. The $f(\theta)$ can be described in Eq. (8) and Eq. (9):

$f(\theta)=b \theta^{2}+c \theta$, for $\theta \leq 15^{\circ}$

$f(\theta)=x \cos ^{2} \theta \sin (\theta)+y \sin ^{2}(\theta)+z$,

for $\theta>15^{\circ}$

Where $b, c, x, y$ and $z$ are the empirical constants. $\theta$ represents the transition angle. Sliding wear dominates if $\theta \leq 15^{\circ}$, and impacting wear dominates if $\theta>15^{\circ}$.

In the erosion model proposed by OKA, the erosion rate is described as:

$E=E_{90}\left(\frac{V}{V_{r e f}}\right)^{k_{2}}\left(\frac{d}{d_{\text {ref }}}\right)^{k_{3}} f(\theta)$

$f(\theta)=(\sin \theta)^{n_{1}}\left(1+H_{v}(1-\sin \theta)\right)^{n_{2}}$

Where E90 is $6.15 \mathrm{e}^{-4}$ for the reference erosion ratio at the impact angle of $90^{\circ} . V$ is the particle impact velocity, $V_{\text {ref }}$ is the reference velocity. $d$ and $d_{r e f}$ is the diameter and reference diameter of particle respectively. $k_{2}$ and $k_{3}$ are the velocity and diameter exponents, respectively, and $f(\theta)$ is the impact angle function. For the condition of sand impact steel, the constant used in the above equations are 


\begin{tabular}{|c|c|c|c|c|c|c|}
\hline$H_{v}$ & $n_{1}$ & $n_{2}$ & $k_{2}$ & $k_{3}$ & $d_{r e f}$ & $V_{\text {ref }}$ \\
\hline $\begin{array}{c}1.8 \\
(\mathrm{GPa})\end{array}$ & 0.8 & 1.3 & 2.35 & 0.19 & $\begin{array}{c}326 \\
(\mu \mathrm{m})\end{array}$ & $\begin{array}{c}104 \\
(\mathrm{~m} / \mathrm{s})\end{array}$ \\
\hline
\end{tabular}

We also adopted the Mclaury model and OKA model respectively in screw centrifugal pump to predict the flow passage components erosion in our previous study. By calculating and deriving the correlation factor functions contained in those two erosion models, the results show that both two erosion models were shown good agreements with the experiment data of the erosion test for volute casing. While the calculated value of the Mclaury model is larger than the measured value of the whole, and the OKA model is smaller than the measured value (Shen et al. 2018). Furthermore, according to the equation of the Mclaury, factors of particle shape are also taken into account in this model, so we decided to use this model in our later work.

Based on the above discussion, the McLaury erosion model was selected to predict the erosion rate of solid particles in water and it has been primarily used in slurry flows which is very close to the condition of centrifugal pump transporting sand laden water.

\subsection{Computational Domain}

The computational domain of double-suction centrifugal pump consists of the following parts, the suction chamber, the double-suction impeller and the half spiral volute casing as show in Fig.8 (a). The computational domain geometric model was generated by the 3D modeling software Pro/e.

\subsection{Mesh Generation}

Model mesh generation were performed using the ICEM, unstructured grid was adopted to discretize the computational domain because of the complex geometry of the double-suction impeller. The surfaces of the blades are key consideration area in the calculation, so the near wall area mesh of blade were refined to better capture the boundary layer flow of the blades. The height of the first layer on the blade surface was set as $0.127 \mathrm{~mm}$, the height ratio was set as 1.1, and the number of the boundary layer was set as 12 . The values of $y^{+}$in the impeller domain were controlled within 100. Figure 8 (b) shows a detailed view of the mesh at the impeller. To confirm that the number of grid cells is adequate, a grid convergence study for the head and efficiency of the pump was performed on 4 different cases as shown in Table 3. Finally, choose the total mesh number of $3,404,088$ for subsequent calculations.

Table 3 Grid convergence study results

\begin{tabular}{|c|c|c|c|c|c|}
\hline $\begin{array}{c}\text { Grid } \\
\text { number }\end{array}$ & $\begin{array}{c}\text { Cell } \\
\text { count }\end{array}$ & $H[\mathrm{~m}]$ & $\varepsilon_{H}[\%]$ & $\eta[\%]$ & $\varepsilon_{\eta}[\%]$ \\
\hline 1 & $\begin{array}{c}1647 \\
891\end{array}$ & 45.75 & 5.10 & 93.57 & 5.33 \\
\hline 2 & $\begin{array}{c}2612 \\
405\end{array}$ & 45.58 & 4.73 & 92.94 & 4.62 \\
\hline 3 & $\begin{array}{c}3404 \\
088\end{array}$ & 45.37 & 4.24 & 92.10 & 3.67 \\
\hline 4 & $\begin{array}{c}3803 \\
873\end{array}$ & 45.49 & 4.51 & 92.64 & 4.28 \\
\hline
\end{tabular}

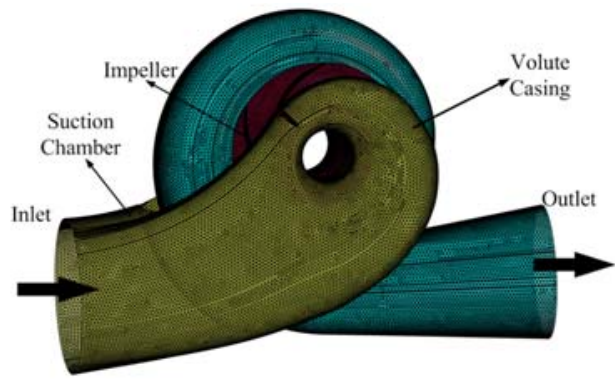

(a)

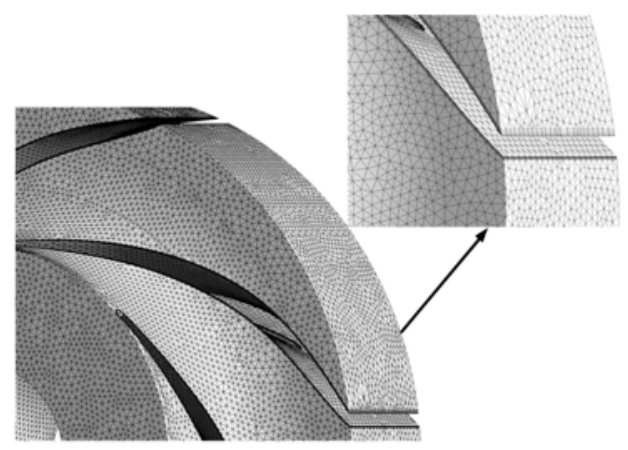

(b)

Fig. 8. Mesh description of computational domain. (a) mesh of double-suction centrifugal pump (b) Mesh details of the impeller.

\subsection{Boundary Conditions and Simulation Procedure}

The velocity inlet is defined in the pump inlet based on the mass flow rate of the pump, particle velocity and liquid velocity are set to the same at the inlet. At the outlet, the outflow boundary condition is imposed. The roughness constant is set to be 0.5 . Boundaries of wall are set as "reflect" and boundaries of inlet and outlet is set as "escape".

In order to improve convergence, the SIMPLEC algorithm is used to solve the governing equations with the second order upwind discretization in momentum, turbulence dissipation rate and turbulence kinetic energy terms (Quan et al. 2019). The liquid phase and the discrete phase convergence criteria is set as $1 \times 10^{-4}$ and in the calculation, The number of continuous phase iterations per DPM iteration is set as 10 . The particle trajectory is calculated based on the Newton's second law, and the discrete random walk (DRW) model is used to predict the dispersion of the particles due to turbulence (Gosman, 1983). The effect of instantaneous turbulent velocity formulations on the particle trajectories were included in this model by stochastic method. The difference between the pump inlet and outlet flow rates of the adjacent step is within $0.001 \%$.

\subsection{Validation of Numerical Modeling}

The validation of the present results has been carried out in 5 different mass flow rate conditions to verify the CFD model adopted in this study. The data of experiment from the pump performance test bed. 


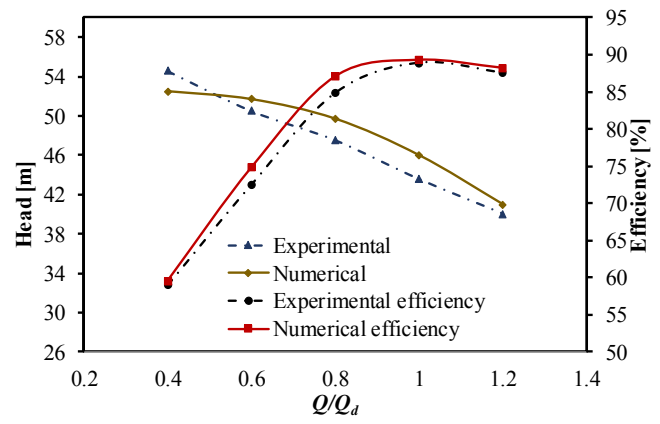

Fig.9. Pump head and efficiency curves.
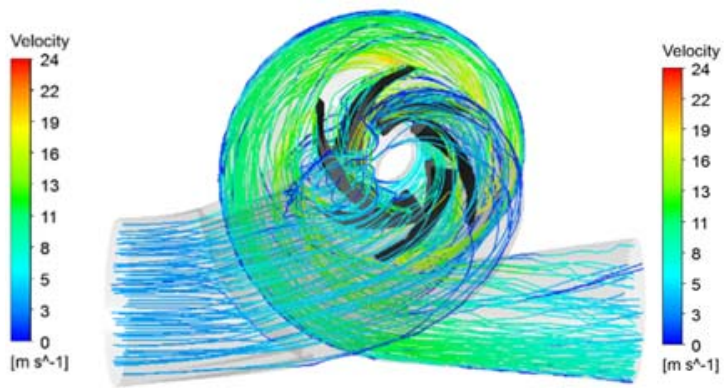

(a)
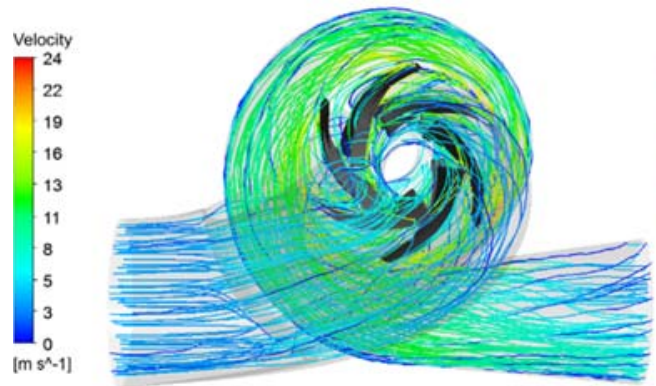

(c)

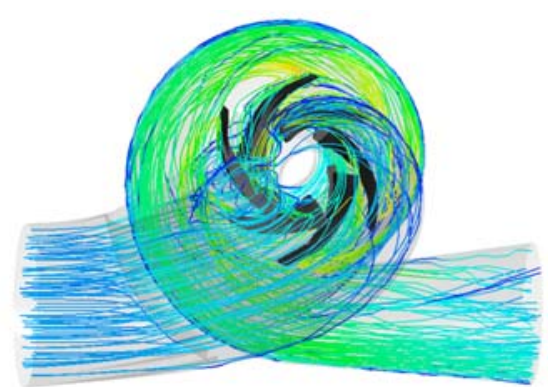

(b)

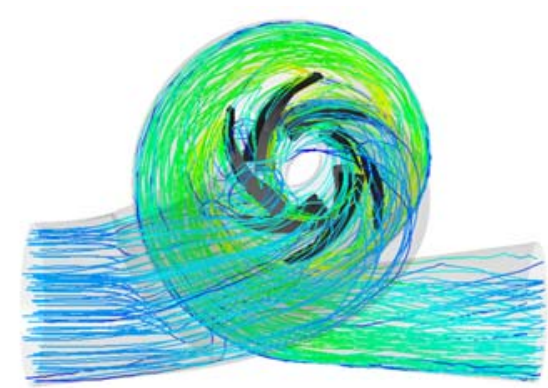

(d)

Fig.10. particles trajectories with different particle size. (a) $4 \mu \mathrm{m}$ (b) $25 \mu \mathrm{m}$ (c) $75 \mu \mathrm{m}$ (d) $100 \mu \mathrm{m}$.

Figure 9 shows the comparison between numerical calculations and test results of pump performance characteristics. The results show that the calculated values of the head and efficiency under the 5 working conditions are basically consistent with the trend of the experimental values. The curves have the same trend and the peaks are at the same operating point. But the calculated values of head and efficiency are slightly higher than the test values expect the working condition of $0.4 Q_{d}$. This is because the volumetric leakage and power loss were not considered in the numerical calculation. The relative error between the calculated value and the experimental value is maintained within $5 \%$, indicating that the numerical calculation method can predict the performance characteristics of the pump accurately.

\section{RESULTS AND DISCUSSIONS}

The variation of particle size and sediment concentration in the Yellow River basin is closely related to geographical location and climatic factors. According to the analysis of modern measured data, the sediment concentration in the Yellow River basin can be changed from below $1 \mathrm{~kg} / \mathrm{m}^{3}$ in non-flood season to over $1000 \mathrm{~kg} / \mathrm{m}^{3}$ in flood season (Yellow River Conservancy Commission of the Ministry of Water Resources Sediment source and its characteristics, 2011), which can be called widerange liquid-sediment two-phase flow. Therefore, it is necessary to study the relationship between blade erosion wear and sediment particle size and sediment concentration.

Many researchers have developed the correlation of the erosion wear and particle parameters (Kazim et al. 1997; Serrano et al. 2018) in previous studies, but still lack of research related to double-suction centrifugal pumps because of its complex structure. The main diameter used in this research are $4 \mu \mathrm{m}, 25$ $\mu \mathrm{m}, 75 \mu \mathrm{m}$ and $100 \mu \mathrm{m}$ according to the particle size distribution data of the middle and upper reaches 

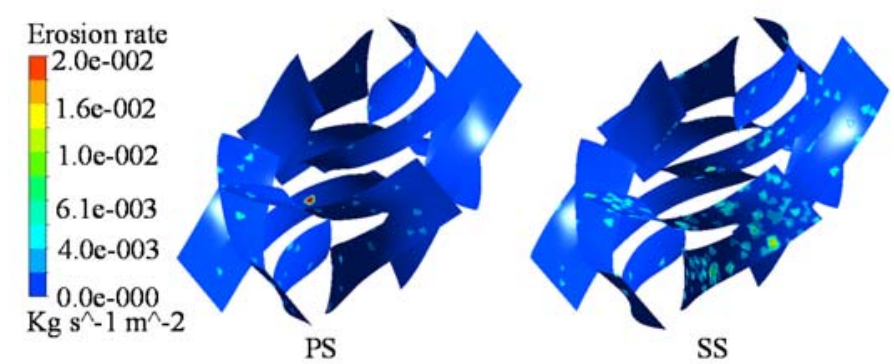

(a)

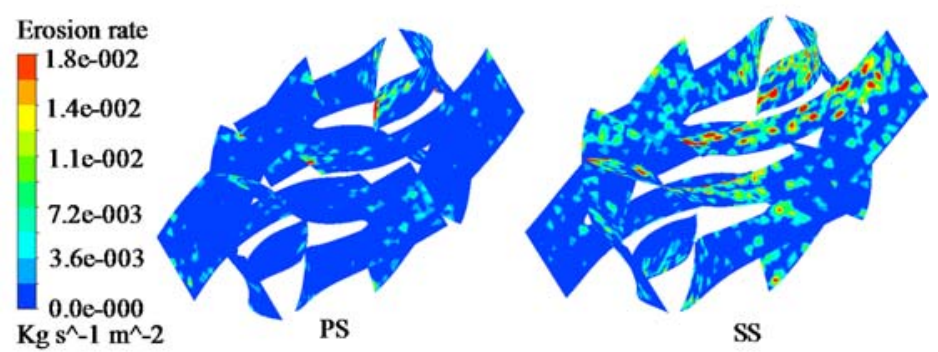

(b)

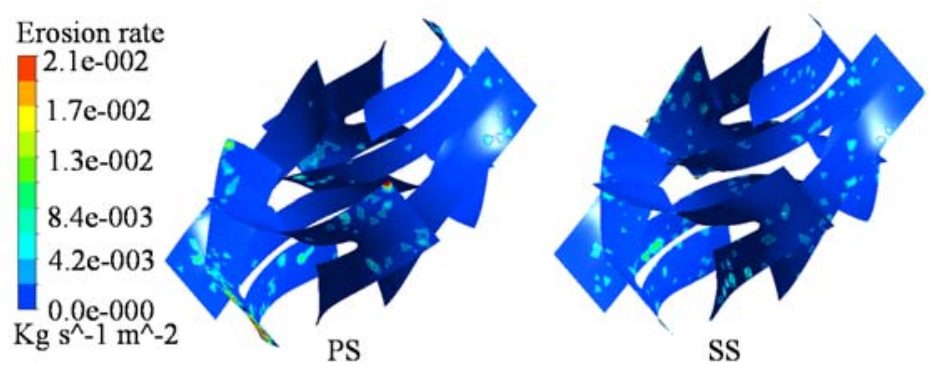

(c)

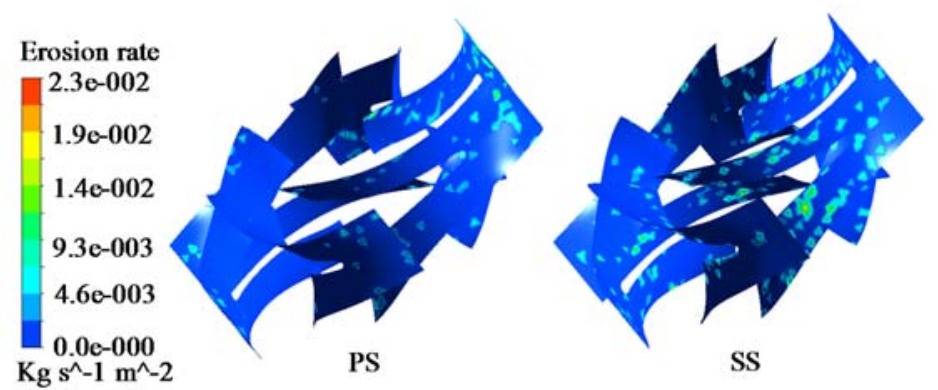

(d)

Fig.11. Blade surfaces erosion rate density distribution. (a) $4 \mu \mathrm{m}$ (b) $25 \mu \mathrm{m}$ (c) $75 \mu \mathrm{m}$ (d) $100 \mu \mathrm{m}$.

of the Yellow River (Shen et al. 2018). The average shape factor of sediment particles in the calculation is set as 0.6 .

\subsection{Effect of Particle Size on Trajectory}

Trajectories of sediment particles in the computational domain are shown in Fig.10. Sediment particles diameter were set to vary from 4 $\mu \mathrm{m}$ to $100 \mu \mathrm{m}$, and the sediment concentration were set as 8,683 PPM during the irrigation period of

\section{JYRIP.}

As shown, the particles enter the pump inlet at a uniform velocity, but different degrees of eddy currents are found around the suction tongue. The velocity of the particles in the computational domain of the impeller is improved, and the particles acquire kinetic energy under the rotation of the blades. The flow at the pump outlet is more turbulent than the inlet flow, because the velocity of sediment particles were greatly improved by the rotation of the 
impeller, when the particle diameter increases from $4 \mu \mathrm{m}$ to $100 \mu \mathrm{m}$, the particle trajectories become more uniform, it is because large particles trend to maintain their own trajectories due to the effect of inertia.

\subsection{Effect of Particle Size and Concentration on Blade Erosion}

The relationship between sediment particle size and the impeller blades erosion was investigated to study the effects of particle diameter on the erosion wear of the blade surfaces. Figure 11 shows the numerical results of the erosion rate density distribution of the pressure side and suction side of the impeller blade. Sediment particles diameter changes from $4 \mu \mathrm{m}$ to $100 \mu \mathrm{m}$ under the same sediment concentration of 8,683 PPM. The results show that with increasing of particle diameter, the erosion wear of blade surfaces is gradually aggravated. In addition, the erosion wear damage of the SS is greater than PS at the same positon of the blade surface.

The change in average erosion rate of impeller blade surfaces with particle diameter at 3 representative sediment particles concentration of $C_{\mathrm{m} 1}=5,074 \mathrm{PPM}$, $C_{\mathrm{m} 2}=8,683 \quad$ PPM and $C_{\mathrm{m} 3}=29,450 \quad$ PPM corresponding to the JYRIP is shown in Fig. 12. Particle diameter ranged from $4 \mu \mathrm{m}$ to $250 \mu \mathrm{m}$, and shape factor was set as 0.6. As shown, the average erosion rate of the blade surfaces increases with increasing of particle diameter. According to the definition of the Stokes number (Peng and Cao. 2016), as the particle diameter increases, stokes number correspondingly increases. Due to the inertia effect, the larger particles are less affected by the fluid drag force, and trend to maintain their own trajectory, impacting with the blade surfaces many times, and resulting in the erosion wear of the blade surfaces with the increase of particle size. However, the slopes decrease with the increase of the particle diameter at a certain sediment concentration. It is because of at the same sediment concentration, an increase particle size means that the number of particles in the same volume is reduced, and the probability of impacting with the blade surfaces is smaller.

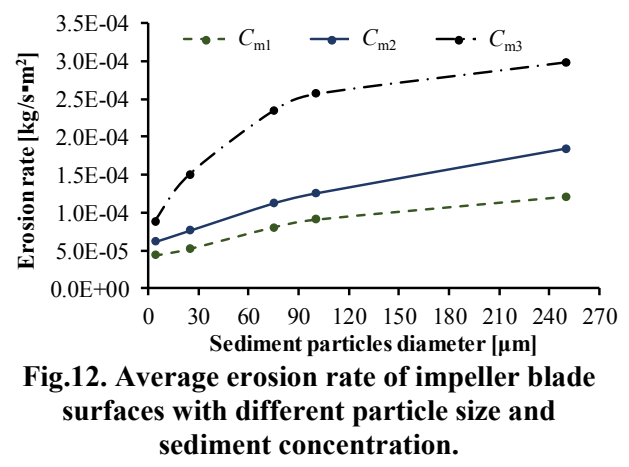

In addition, the average erosion rate of the impeller surfaces increases with increasing sediment concentration. Meaning that the greater the sediment concentration, the greater the probability of contact between particles and blade surfaces, and the more collisions, the more the blade erosion wear will be aggravated.

\section{CONCLUSIONS}

The regular operation observation and morphology of double-suction centrifugal pump impeller were studied to investigate erosion wear characteristics at different regions. The effects of particle size and concentration on the trajectories of sediment particles and the erosion of impeller blades were also studied by numerical simulations. Numerical results are in good agreement with the experimental results. From the present research, the following conclusions can be drawn:

(1) In the direction from the LE to the TE, the surface wear shape of the blade changes significantly from circular shape to strip shape, and the wear damage area increases correspondingly.

(2) Large particle size particles tend to maintain their own trajectory motion and cause more severe erosion wear damage. Under the same sediment concentration, after the particle size increases from $100 \mu \mathrm{m}$ to $250 \mu \mathrm{m}$, the wear damage tends to be slowly.

(3) Erosion rate increases with the sediment concentration increase from 5,074 PPM to $29,450 \mathrm{PPM}$. And the erosion rate of the SS is greater than PS at the same positon of the blade surface.

\section{ACKNOWLEDGMENTS}

The work was supported by the National Natural Science Foundations (Grant No. 51536006), and Natural Science Foundation of Gansu province (Grant No. 1606RJYA297). Shaanxi Provincial Natural Science Basic Research Project (2019JLM58) and Shaanxi Water Conservancy Science and Technology Project (2019slkj-15)

\section{REFERENCES}

Ahlert, K. R. (1994). Effects of particle impingement angle and surface wetting on solid particle erosion of AISI 1018 steel $\mathrm{Ph}$. D. thesis, The University of Tulsa.

ANSYS FLUENT 12.0/12.1 Documentation, ANSYS inc. Southpoint, 275 Technology drive, Canonburg, PA 15317, USA.

Bai, L., L. Zhou, C. Han, Y. Zhu and W. Shi (2019). Numerical Study of Pressure Fluctuation and Unsteady Flow in a Centrifugal Pump. Processes 7(6), 354.

Bai, L., L. Zhou, X. Jiang, Q. Pang and D. Ye (2019). Vibration in a Multistage Centrifugal Pump under Varied Conditions. Shock and Vibration 
$1-9$.

Bitter, J. G. A. (1963). A study of erosion phenomena part I. wear 6(1), 5-21.

Brown, G. J. (2002). Erosion prediction in slurry pipeline tee-junctions. Applied mathematical modelling 26(2), 155-170.

Chitrakar, S., H. P. Neopane and O. G. Dahlhaug (2016). Study of the simultaneous effects of secondary flow and sediment erosion in Francis turbines. Renewable Energy 97, 881891.

Coutier-Delgosha, O., R. Fortes-Patella, J. L. Reboud, M. Hofmann and B. Stoffel (2003). Experimental and numerical studies in a centrifugal pump with two-dimensional curved blades in cavitating condition. Journal of Fluids Engineering 125(6), 970-978.

Finnie, I. (1960). Erosion of surfaces by solid particles. Wear 3(2), 87-103.

Gosman, A. D. and E. Loannides (1983). Aspects of computer simulation of liquid-fueled combustors. Journal of energy 7(6), 482-490.

Grant, G. and W. Tabakoff (1975). Erosion prediction in turbomachinery resulting from environmental solid particles. Journal of Aircraft 12(5), 471-478.

Haider, A. and O. Levenspiel (1989). Drag coefficient and terminal velocity of spherical and nonspherical particles. Powder technology 58(1), 63-70.

Hashish, M. (1988). An improved model of erosion by solid particles. In Proceedings of the 7th International Conference on Erosion by liquid and solid impact. (pp 66/1-66/9). ELSI VII, Cambridge, England.

Hirschi, R., P. Dupont, F. Avellan, J. N. Favre, J. F. Guelich and E. Parkinson (1998). Centrifugal pump performance drop due to leading edge cavitation: numerical predictions compared with model tests. Journal of Fluids Engineering 120, 705-711.

Kazim, K. A., B. Maiti and P. Chand (1997). A correlation to predict the performance characteristics of centrifugal pumps handling slurries. Proceedings of the Institution of Mechanical Engineers, Part A: Journal of Power and Energy 211(2), 147-157.

Liu, Z. G., S. Wan, V. B. Nguyen and Y. W. Zhang (2014). A numerical study on the effect of particle shape on the erosion of ductile materials. Wear 313(1-2), 135-142.

Lu, J. X. (1999). Silt abrasion of water pump and its countermeasures. Journal of drainage irrigation mechanical engineering 1,20-23.

McLaury, B. S. (1996). Predicting Solid Particle Erosion Resulting From Turbulent Fluctuations in Oilfield Geometries. Ph.D. dissertation, Department of Mechanical
Engineering. The University of Tulsa, Tulsa, OK.

Menter, F. R. (1994). Two-equation eddy-viscosity turbulence models for engineering applications. AIAA journal 32(8), 1598-1605.

Nguyen, V. B., Q. B. Nguyen, Y. W. Zhang, C. Y. H. Lim and B. C. Khoo (2016). Effect of particle size on erosion characteristics. Wear $348,126-$ 137.

Oka, Y. I. and T. Yoshida (2005). Practical estimation of erosion damage caused by solid particle impact: Part 2: Mechanical properties of materials directly associated with erosion damage. Wear 259(1-6), 102-109.

Parsi, M., M. Kara, M. Agrawal, N. Kesana, A. Jatale, P. Sharma and S. Shirazi (2017). CFD simulation of sand particle erosion under multiphase flow conditions. Wear 376, 11761184

Peng, W. and X. Cao (2016). Numerical simulation of solid particle erosion in pipe bends for liquid-solid flow. Powder technology 294, 266-279.

Quan, H., J. Li, R. Li, G. Shi, Q. Su and B. Fu (2017). Mathematical modeling for the evolution of the large-and meso-scale vortex in the screw centrifugal pump with the buoyancy effect. Advances in Mechanical Engineering 9(5), 112.

Quan, H., Y. Chai, R. Li and J. Guo (2019). Numerical simulation and experiment for study on internal flow pattern of vortex pump. Engineering Computations 36(5), 1579-1596.

Serrano, R. O. P., L. P. Santos, E. M. de Faria Viana, M. A. Pinto and C. B. Martinez (2018). Case study: Effects of sediment concentration on the wear of fluvial water pump impellers on Brazil's Acre River. Wear, 408, 131-137.

Shen, Z. and W. Chu (2018, November). Effect of Particle Parameters on Erosion Wear and Performance of Screw Centrifugal Pump. In ASME 2018 International Mechanical Engineering Congress and Exposition (pp. V007T09A018-V007T09A018). American Society of Mechanical Engineers.

Shen, Z., W. Chu and W. Dong (2018). Effect of particle parameters on flow field and erosion wear characteristics of flow passage components in screw centrifugal pump. Transactions of the Chinese Society of Agricultural Engineering (Transactions of the CSAE) 34(6), 58 - 66.

Shen, Z., W. Chu, X. Li and W. Dong (2019). Sediment erosion in the impeller of a doublesuction centrifugal pump-A case study of the Jingtai Yellow River Irrigation Project, China. Wear 422, 269-279.

Stachowiak, G. and A. W. Batchelor (2013). Engineering tribology. Butterworth- 
Z. Shen et al. / JAFM, Vol. 13, No. 4, pp. 1131-1142, 2020.

Heinemann.

Van der Schoot, M. and F. Visser (2015, July). Efficiency upgrade of a double-case pump using CFD-based design optimization and scaled model tests. In ASME/JSME/KSME 2015 Joint Fluids Engineering Conference. American Society of Mechanical Engineers Digital Collection.

Wakeman, T. and W. Tabakoff (1982). Measured particle rebound characteristics useful for erosion prediction. In ASME 1982 International Gas Turbine Conference and Exhibit (pp. V003T05A005-V003T05A005). American Society of Mechanical Engineers.

Wang, L., H. Liu, K. Wang, L. Zhou, X. Jiang and Y. $\operatorname{Li}(2019)$. Numerical simulation of the sound field of a five-stage centrifugal pump with different turbulence models. Water 11(9), 1777.

Wilson, K. C., G. R. Addie, A. Sellgren and R. Clift (2006). Slurry transport using centrifugal pumps. Springer Science \& Business Media.
Yellow River Conservancy Commission of the Ministry of Water Resources Sediment source and its characteristics. [Online]. /http://www.yrcc.gov.cn/hhyl/hhgk/hs/ns /201108/t20110814_103396.html).

Zhao, Y. P. (2009). Wear failure analysis of impeller blade of single-stage double-suction centrifugal pump. Gansu water conserve hydropower Technology 45 (4), 30-31.

Zhu, H., J. Zhu, R. Rutter and H. Q. Zhang (2019). A Numerical Study on Erosion Model Selection and Effect of Pump Type and Sand Characters in Electrical Submersible Pumps by Sandy Flow. Journal of Energy Resources Technology 141(12),122004-1-122004-15.

Zhu, H., J. Zhu, Z. Zhou, R. Rutter, M. Forsberg, S. Gunter and H. Q. Zhang (2019, March 22). Experimental Study of Sand Erosion in Multistage Electrical Submersible Pump ESP: Performance Degradation, Wear and Vibration. International Petroleum Technology Conference, 395-409. 